Old Dominion University ODU Digital Commons

1994

\title{
A Fast Numerical Solution of Scattering by a Cylinder: Spectral Method for the Boundary Integral Equations
}

Fang Q.Hu

Old Dominion University, fhu@odu.edu

Follow this and additional works at: https://digitalcommons.odu.edu/mathstat_fac_pubs

Part of the Partial Differential Equations Commons, and the Physics Commons

\section{Repository Citation}

Hu, Fang Q. "A Fast Numerical Solution of Scattering by a Cylinder: Spectral Method for the Boundary Integral Equations" (1994). Mathematics \& Statistics Faculty Publications. 22.

https://digitalcommons.odu.edu/mathstat_fac_pubs/22

\section{Original Publication Citation}

Hu, F. Q. (1994). A fast numerical solution of scattering by a cylinder: Spectral method for the boundary integral equations. Journal of the Acoustical Society of America, 96(6), 3693-3703. doi:10.1121/1.410559 
A fast numerical solution of scattering by a cylinder: Spectral method for the boundary integral equations

Fang Q. Hu

Citation: The Journal of the Acoustical Society of America 96, 3693 (1994); doi: 10.1121/1.410559

View online: http://dx.doi.org/10.1121/1.410559

View Table of Contents: http://asa.scitation.org/toc/jas/96/6

Published by the Acoustical Society of America 


\title{
A fast numerical solution of scattering by a cylinder: Spectral method for the boundary integral equations
}

\author{
Fang Q. Hu \\ Department of Mathematics and Statistics, Old Dominion University, Norfolk, Virginia 23529
}

(Received 16 March 1994; accepted for publication 9 July 1994)

\begin{abstract}
It is known that the exact analytic solutions of wave scattering by a circular cylinder, when they exist, are not in a closed form but in infinite series which converge slowly for high frequency waves. In this paper, a fast numerical solution is presented for the scattering problem in which the boundary integral equations, reformulated from the Helmholtz equation, are solved using a Fourier spectral method. It is shown that the special geometry considered here allows the implementation of the spectral method to be simple and very efficient. The present method differs from previous approaches in that the singularities of the integral kernels are removed and dealt with accurately. The proposed method preserves the spectral accuracy and is shown to have an exponential rate of convergence. Aspects of efficient implementation using FFT are discussed. Moreover, the boundary integral equations of combined single- and double-layer representation are used in the present paper. This ensures the uniqueness of the numerical solution for the scattering problem at all frequencies. Although a strongly singular kernel is encountered for the Neumann boundary conditions, it is shown that the hypersingularity can be handled easily in the spectral method. Numerical examples that demonstrate the validity of the method are also presented.
\end{abstract}

PACS numbers: 43.20.Fn

\section{INTRODUCTION}

The exact analytic solutions of wave scattering by a circular cylinder, obtainable for simple incident waves, are not in a closed form but in infinite series of Bessel and Hankel functions of increasing orders. Such solutions converge slowly, especially for high frequency waves, which render their numerical evaluation inefficient. This paper presents a fast numerical solution of wave scattering that only requires the computation of Bessel and Hankel functions of order zero. Furthermore, the numerical solution is valid for any form of the incident waves of all frequencies.

When developing numerical solutions, wave scattering problems are often conveniently formulated in boundary integral equations (BIE). ${ }^{1}$ The advantages of the boundary integral equation method (BIEM) include reducing the dimension of the problem and transforming an infinite domain to finite boundaries in which the far-field radiation condition is satisfied automatically. The Boundary Integral Equations are commonly solved computationally by the boundary element methods (BEM). ${ }^{2}$ In this method, the boundary is divided into finite elements and integrations over each boundary element are approximated by quadratures, e.g., the linear elements.

In this paper, we develop a spectral method of solving the boundary integral equations, reformulated from the Helmholtz equation, for numerical solutions of wave scattering by a circular cylinder. Previously, for this special geometry, a "fast numerical method" based on the Fourier approximations has been formulated by Bojarski, ${ }^{3}$ who pointed out that the boundary integral equation of wave scattering can be solved easily and efficiently in the Fourier spectrum domain of the solution. Due to the simplicity of the geometry, an explicit relation between the Fourier coefficients of the solution and those of the boundary condition was found. It was argued that the numerical approach was more efficient than directly evaluating the infinite series of the exact solutions. Indeed, the exact solutions contain Bessel and Hankel functions of higher orders whose numerical evaluation is more difficult and costly as the order increases. Recently, a similar approach has been used and extended by Schuster ${ }^{4}$ for a wave transmission problem of concentric cylinders.

In the present paper, we point out that the numerical formulations given previously are not achieving the optimal accuracy of the Fourier spectral methods. It is known that, although any periodic function can be approximated by a truncated Fourier series, the rate of convergence of such an approximation depends on its smoothness. Unfortunately, the integral kernels for the Helmholtz equation are not smooth. In particular, the 2D Green's function of the Helmholtz equation, appearing in the integral equations, possesses a logarithmic singularity. Furthermore, the normal derivative of the Green's function also contains a term involving the logarithmic function. The nonsmoothness of the integral kernels, however, was not explicitly treated in the previous formulations. It will be seen that it is critical to remove the nonsmoothness of the integral kernels in order to achieve fast convergence in the Fourier spectral formulation. By a proper treatment of the singularities, the present numerical formulation yields accurate solutions with significantly fewer datum points. Moreover, the boundary integral equations of combined single- and double-layer representation are used in the present paper. This ensures the uniqueness of the numerical solution for the scattering problem at all frequencies. ${ }^{1,5} \mathrm{Al}$ though a combined layer formulation results in a strongly singular kernel for the Neumann boundary conditions, we show that the hypersingularity is handled easily in the spectral method. 
In the next section, the formulations of the boundary integral equations for wave scattering problems are given. Then, in Secs. II and III, the Fourier spectral methods for the Dirichlet and Neumann boundary conditions are presented. Numerical results are shown in Sec. IV. Section V contains the conclusions. Some analytic results are also given in the Appendix.

\section{BOUNDARY INTEGRAL EQUATIONS}

Let us consider wave scattering by a circular cylinder $\Gamma$ of radius $a$. The wave equation for the scattered function $\phi$ with assumed time dependency of $e^{-i \omega t}$ is reduced to the Helmholtz equation

$$
\nabla^{2} \phi+\kappa^{2} \phi=0
$$

where $\kappa=\omega / c$ ( $c$ is the wave speed) and $\nabla^{2}$ is the 2D Laplace operator $\nabla^{2}=\partial^{2} / \partial x^{2}+\partial^{2} / \partial y^{2}$. The boundary condition considered in this paper will be one of the following types

$$
\text { Dirichlet (soft): } \phi(\mathbf{r})=b(\mathbf{r}) \text { on } \Gamma
$$

or

$$
\text { Neumann (hard): } \frac{\partial \phi}{\partial \mathbf{n}}(\mathbf{r})=b(\mathbf{r}) \text { on } \Gamma \text {. }
$$

The Helmholtz equation (1) together with the boundary condition can be reformulated into a boundary integral equation. This can be done in various ways. ${ }^{1,5}$ For scattering problems considered in the present paper, we use a combination of single- and double-layer formulation in which the solution $\phi$ at any point $\mathbf{r}^{\prime}$ in the scattered field is represented by an integral on the boundary as ${ }^{5}$

$$
\phi\left(\mathbf{r}^{\prime}\right)=\int_{\Gamma}\left(\frac{\partial G}{\partial \mathbf{n}}-i \eta G\right) f(\mathbf{r}) d \Gamma,
$$

where $\eta$ is any real number such that

$$
\eta \operatorname{Re}(\kappa)>0 \text {. }
$$

The use of a combined formulation ensures the uniqueness of the numerical solution for exterior problems. ${ }^{1,5}$ In $(2), f(\mathbf{r})$ is an unknown layer distribution function and the Green's function $G\left(\mathbf{r}, \mathbf{r}^{\prime}\right)$, whose form will be given later, satisfies the following equation

$$
\nabla^{2} G+\kappa^{2} G=-\delta\left(\mathbf{r}-\mathbf{r}^{\prime}\right)
$$

Here the normal derivative $\partial / \partial \mathrm{n}$ is assumed to be taken in the direction outward from the cylinder.

The boundary integral equation associated with the layer representation (2) is 5

$$
\frac{1}{2} f\left(\mathbf{r}_{\Gamma}^{\prime}\right)+\int_{\Gamma}\left(\frac{\partial G}{\partial \mathbf{n}}-i \eta G\right) f\left(\mathbf{r}_{\Gamma}\right) d \Gamma=b\left(\mathbf{r}_{\Gamma}^{\prime}\right)
$$

for Dirichlet boundary conditions and

$$
\frac{i \eta}{2} f\left(\mathbf{r}_{\Gamma}^{\prime}\right)+\int_{\Gamma}\left(\frac{\partial^{2} G}{\partial \mathbf{n}^{\prime} \partial \mathbf{n}}-i \eta \frac{\partial G}{\partial \mathbf{n}^{\prime}}\right) f\left(\mathbf{r}_{\Gamma}\right) d \Gamma=b\left(\mathbf{r}_{\Gamma}^{\prime}\right)
$$

for Neumann boundary conditions, respectively. In (4a) and (4b), $\mathbf{r}_{\Gamma}$ denotes the boundary points. After the layer distri- bution function $f$ has been solved from the integral equation (4a) or (4b), the solution of the Helmholtz equation $\phi$ is found by the boundary integral (2).

Now for a circular cylinder of radius $a$, the boundary contour can be expressed as

$$
\mathbf{r}_{\Gamma}(\theta)=(a \cos \theta, a \sin \theta), \quad 0 \leqslant \theta \leqslant 2 \pi .
$$

The normal vector to be used in (4a) and (4b) is $\mathbf{n}=(\cos \theta, \sin \theta)$.

The Green's function and its normal derivative are ${ }^{5,6}$

$$
\begin{aligned}
G & =\frac{i}{4} H_{0}^{(1)}\left(\kappa\left|\mathbf{r}_{\Gamma}(\theta)-\mathbf{r}_{\Gamma}\left(\theta^{\prime}\right)\right|\right) \\
& =\frac{i}{4} H_{0}^{(1)}\left(2 \kappa a\left|\sin \frac{\theta-\theta^{\prime}}{2}\right|\right),
\end{aligned}
$$

and

$$
\begin{aligned}
\frac{\partial G}{\partial \mathbf{n}} & =-\frac{i \kappa}{4} H_{1}^{(1)}\left(\kappa\left|\mathbf{r}_{\Gamma}(\theta)-\mathbf{r}_{\Gamma}\left(\theta^{\prime}\right)\right|\right) \frac{\left(\mathbf{r}_{\Gamma}(\theta)-\mathbf{r}_{\Gamma}\left(\theta^{\prime}\right)\right) \cdot \mathbf{n}}{\left|\mathbf{r}_{\Gamma}(\theta)-\mathbf{r}_{\Gamma}\left(\theta^{\prime}\right)\right|} \\
& =-\frac{i \kappa}{4} H_{1}^{(1)}\left(2 \kappa a\left|\sin \frac{\theta-\theta^{\prime}}{2}\right|\right)\left|\sin \frac{\theta-\theta^{\prime}}{2}\right|
\end{aligned}
$$

in which we have used the fact that $\left|\mathbf{r}_{\Gamma}(\theta)-\mathbf{r}_{\Gamma}\left(\theta^{\prime}\right)\right|=2 a\left|\sin \left(\theta-\theta^{\prime}\right) / 2\right|$.

It is important to note here that $G$ and $\partial G / \partial n$ are functions of $\theta-\theta^{\prime}$. As will be seen later, this allows the implementation of the Fourier spectral method to take a simple form.

Thus we express the boundary integral equation (4a) for the Dirichlet boundary conditions as

$$
\begin{aligned}
& \frac{1}{2} f\left(\theta^{\prime}\right)+\int_{0}^{2 \pi}\left(\frac{\partial G}{\partial \mathbf{n}}\left(\theta-\theta^{\prime}\right)-i \eta G\left(\theta-\theta^{\prime}\right)\right) f(\theta) a d \theta \\
& =b\left(\theta^{\prime}\right)
\end{aligned}
$$

and Eq. (4b) for the Neumann boundary conditions as

$$
\begin{aligned}
& \frac{i \eta}{2} f\left(\theta^{\prime}\right)+\int_{0}^{2 \pi}\left(\frac{\partial^{2} G}{\partial \mathbf{n}^{\prime} \partial \mathbf{n}}\left(\theta-\theta^{\prime}\right)-i \eta \frac{\partial G}{\partial \mathbf{n}^{\prime}}\left(\theta-\theta^{\prime}\right)\right) \\
& \times f(\theta) a d \theta=b\left(\theta^{\prime}\right) .
\end{aligned}
$$

For clarity, the dependencies on $\theta$ and $\theta^{\prime}$ have been expressed explicitly in (8a) and (8b).

In the next two sections, we give the numerical formulations of solving the integral equations (8a) and ( $8 b)$ by a Fourier spectral method. Since different types of singularities are encountered, the two equations will be dealt with separately.

\section{SPECTRAL METHOD FOR DIRICHLET BOUNDARY CONDITIONS}

\section{A. Formulation}

Let the layer distribution function $f(\theta)$ and the boundary condition $b(\theta)$ be approximated by the truncated Fourier series as 


$$
\begin{aligned}
& f(\theta)=\sum_{n=-N / 2}^{N / 2-1} f_{n} e^{i n \theta}, \\
& b(\theta)=\sum_{n=-N / 2}^{N / 2-1} b_{n} e^{i n \theta},
\end{aligned}
$$

where $b_{n}$ are obtained by the FFT from the prescribed boundary condition and $f_{n}$ are the unknown coefficients. In (9) and (10), the particular form of truncated Fourier series has been taken for the convenience of applying FFT programs.

Substituting (9) and (10) into the boundary integral equation for the Dirichlet boundary conditions (8a), we get

$$
\begin{array}{r}
\frac{1}{2} \sum_{n=-N / 2}^{N / 2-1} f_{n} e^{i n \theta^{\prime}}+\sum_{n=-N / 2}^{N / 2-1}\left[f _ { n } \int _ { 0 } ^ { 2 \pi } \left(\frac{\partial G}{\partial \mathbf{n}}\left(\theta-\theta^{\prime}\right)\right.\right. \\
\left.\left.-i \eta G\left(\theta-\theta^{\prime}\right)\right) e^{i n \theta} a d \theta\right]=\sum_{n=-N / 2}^{N / 2-1} b_{n} e^{i n \theta^{\prime}}
\end{array}
$$

For simplicity, let

$$
x=\theta-\theta^{\prime} .
$$

By equating the coefficients of $e^{i n \theta^{\prime}}$, Eq. (11) is easily reduced to

$$
\frac{1}{2} f_{n}+f_{n} \int_{0}^{2 \pi}\left(\frac{\partial G}{\partial \mathbf{n}}(x)-i \eta G(x)\right) e^{i n x} a d x=b_{n},
$$

for $-N / 2 \leqslant n \leqslant N / 2-1$.

It is seen that the integrals appearing in (12) are related to the Fourier coefficients of $(\partial G / \partial \mathbf{n})(x)$ and $G(x)$. From (6) and (7), it is also clear that both are periodic functions of $x$, with a period of $2 \pi$. Thus, if we let $G(x)$ and $(\partial G / \partial \mathrm{n})(x)$ be approximated by truncated Fourier series as

$$
\begin{aligned}
& G(x)=\sum_{n=-N / 2}^{N / 2-1} g_{n} e^{-i n x}, \\
& \frac{\partial G}{\partial \mathbf{n}}(x)=\sum_{n=-N / 2}^{N / 2-1} h_{n} e^{-i n x},
\end{aligned}
$$

then, the integral in (12) equals to $2 \pi a\left(h_{n}-i \eta g_{n}\right)$. It follows that

$$
\frac{1}{2} f_{n}+2 \pi a f_{n}\left(h_{n}-i \eta g_{n}\right)=b_{n} .
$$

Therefore, the Fourier coefficients of the layer distribution function $f(\theta)$ are obtained explicitly as

$$
f_{n}=\frac{b_{n}}{\frac{1}{2}+2 \pi a\left(h_{n}-i \eta g_{n}\right)} .
$$

The above equation shows that once the Fourier coefficients of $G(x)$ and $(\partial G / \partial \mathrm{n})(x)$ have been found, the layer distribution function $f(\theta)$ is known immediately.

Actually, the Fourier coefficients of $G(x)$ and $(\partial G /$ $\partial n)(x)$ can be found in exact form using higher-order Bessel and Hankel functions. They are derived in Appendix A.
Nonetheless, the numerical evaluation of the exact expressions becomes more ineffective and costly as the order of the special functions increases. In what follows we give the numerical method that computes the Fourier coefficients $g_{n}$ and $h_{n}$ accurately and efficiently.

\section{B. Computation of $g_{n}$ and $h_{n}$}

In general, the Fourier coefficients of a periodic function can be obtained efficiently by using a fast Fourier transform algorithm (FFT). However, the accuracy of the Fourier coefficients computed by the FFT using a given number of datum points depends on the smoothness of the function. Only when the function is infinitely smooth (i.e., infinitely differentiable), the error of Fourier coefficients computed by FFT decays faster than any power of $1 / N$, where $N$ is the number of datum points. Such a convergence is often referred to as an exponential convergence and the method is said to have spectral accuracy. ${ }^{7,8}$ Our aim here is to compute $g_{n}$ and $h_{n}$ by the FFT with spectral accuracy even though the functions $G$ and $\partial G / \partial$ are not smooth.

In the numerical approaches proposed previously, ${ }^{3,4}$ the Fourier coefficients $g_{n}$ and $h_{n}$ were computed directly as the FFT of the $G(x)$ and $(\partial G / \partial n)(x)$, respectively. However, the Green's function $G(x)$ has a logarithmic singularity at $x=0$, where $\theta=\theta^{\prime}$, due to the Hankel function of order zero in (6), and its Fourier series converges at the rate of $1 / N$. Thus, direct computation of $g_{n}$ from $G(x)$ using FFT yields results whose accuracy is only comparable to a first-order method. Furthermore, the function $(\partial G / \partial n)(x)$ also has a nonsmooth derivative at $x=0$, and its Fourier series converges at the rate of $1 / N^{3}$. Thus direct computation of $h_{n}$ from $(\partial G / \partial \mathrm{m})(x)$ is only comparable to a third order method. Alternatively, as will be shown below, by properly treating the nonsmoothness of $G(x)$ and $(\partial G / \partial \mathbf{n})(x), g_{n}$, and $h_{n}$ are computed with spectral accuracy.

To examine the singularity of $G(x)$, we note that

$$
\begin{aligned}
G(x) & =\frac{i}{4} H_{0}^{(1)}\left(2 \kappa a\left|\sin \frac{x}{2}\right|\right) \\
& =\frac{i}{4}\left[J_{0}\left(2 \kappa a\left|\sin \frac{x}{2}\right|\right)+i Y_{0}\left(2 \kappa a\left|\sin \frac{x}{2}\right|\right)\right],
\end{aligned}
$$

in which $J_{0}$ and $Y_{0}$ are the zeroth order Bessel functions of the first and second kind, respectively. Using the asymptotic series for small arguments, we have ${ }^{9}$

$$
\begin{aligned}
& J_{0}(z)=1-\frac{z^{2}}{4}+\frac{z^{4}}{64}-\cdots, \\
& Y_{0}(z)=\frac{2}{\pi} \ln \left(\frac{z}{2}\right) J_{0}(z)+\frac{2 \gamma}{\pi} J_{0}(z)+\frac{z^{2}}{2 \pi}-\cdots .
\end{aligned}
$$

It follows that, for $|x|$ small

$$
\begin{aligned}
G(x)= & -\frac{1}{2 \pi} \ln \left(\kappa a\left|\sin \frac{x}{2}\right|\right) J_{0}\left(2 \kappa a\left|\sin \frac{x}{2}\right|\right)-\frac{\gamma}{2 \pi}+\frac{i}{4} \\
& +O\left(x^{2}\right),
\end{aligned}
$$

in which $O\left(x^{2}\right)$ represents a power series in $x^{2}$, and $\gamma$ is the Euler's constant, $\gamma=0.577215 \ldots$. To compute the Fourier 
coefficients of $G(x)$ efficiently and accurately, we note that the Fourier series of the logarithmic periodic function $\ln (\kappa a|\sin x / 2|)$ in $(17)$ is ${ }^{6}$

$$
\ln \left(\kappa a\left|\sin \frac{x}{2}\right|\right)=\ln \left(\frac{\kappa a}{2}\right)-\sum_{n=1}^{\infty} \frac{\cos (n x)}{n} .
$$

Thus, we can "subtract out" the singularity in $G(x)$ by forming

$$
\begin{aligned}
\bar{G}(x)= & \frac{i}{4} H_{0}^{(1)}\left(2 \kappa a\left|\sin \frac{x}{2}\right|\right) \\
& +\frac{1}{2 \pi} \ln \left(\kappa a\left|\sin \frac{x}{2}\right|\right) J_{0}\left(2 \kappa a\left|\sin \frac{x}{2}\right|\right)
\end{aligned}
$$

and then writing the Green's function as

$$
G(x)=\bar{G}(x)-\frac{1}{2 \pi} \ln \left(\kappa a\left|\sin \frac{x}{2}\right|\right) J_{0}\left(2 \kappa a\left|\sin \frac{x}{2}\right|\right) .
$$

It is easy to see that $\bar{G}(x)$ is finite for all values of $x$. Furthermore, both $\bar{G}(x)$ and $J_{0}(2 \kappa a|\sin x / 2|)$ in (19a) are periodic and infinitely differentiable. Thus, their Fourier coefficients can be computed with spectral accuracy using FFT. The Fourier coefficients of the Green's function $G(x), g_{n}$, will be computed according to (19a) where the term involving the logarithmic function is computed by using convolution sums.

We now study the nonsmoothness of the normal derivative of the Green's function $(\partial G / \partial n)(x)$. The asymptotic series of the Bessel functions of first order for small argument are $^{9}$

$$
\begin{aligned}
& J_{1}(z)=\frac{z}{2}-\frac{z^{3}}{16}+\cdots \\
& Y_{1}(z)=-\frac{2}{\pi z}+\frac{2}{\pi} \ln \left(\frac{z}{2}\right) J_{1}(z)+\frac{2 \gamma-1}{2 \pi} z-\cdots
\end{aligned}
$$

Then

$$
\begin{aligned}
\frac{\partial G}{\partial \mathbf{n}}(x)= & -\frac{i \kappa}{4} H_{1}^{(1)}\left(2 \kappa a\left|\sin \frac{x}{2}\right|\right)\left|\sin \frac{x}{2}\right| \\
= & -\frac{i \kappa}{4}\left[J_{1}\left(2 \kappa a\left|\sin \frac{x}{2}\right|\right)\right. \\
& \left.+i Y_{1}\left(2 \kappa a\left|\sin \frac{x}{2}\right|\right)\right]\left|\sin \frac{x}{2}\right| \\
= & -\frac{1}{4 \pi a}+\frac{\kappa}{2 \pi} \ln \left(\kappa a\left|\sin \frac{x}{2}\right|\right) J_{1}\left(2 \kappa a\left|\sin \frac{x}{2}\right|\right) \\
& \times\left|\sin \frac{x}{2}\right|+O\left(x^{2}\right) .
\end{aligned}
$$

Thus although $\partial G / \partial \mathrm{n}$ is a finite function, due to the logarithmic function appearing in the second term shown in
(20), it does not have a smooth second derivative at $x=0$. For this reason, its Fourier approximation will converge only at the rate of $1 / N^{3}$.

The Fourier coefficients of $\partial G / \partial \mathrm{n}$, however, can be found easily using the relation to $g_{n}$ given in the Appendix. In particular, we have

$$
h_{n}=\left\{\begin{array}{l}
-\frac{\kappa^{2} a}{4 n}\left(g_{n+1}-g_{n-1}\right), \quad n \neq 0,-\frac{N}{2}, \frac{N}{2}-1, \\
\frac{\kappa^{2} a}{4}\left(g_{2}-g_{0}\right)-\frac{1}{a} g_{1}, \quad n=0, \\
-\frac{\kappa^{2} a}{4 n} g_{-N / 2+1}, \quad n=-\frac{N}{2}, \\
\frac{\kappa^{2} a}{4 n} g_{N / 2-2}, \quad n=\frac{N}{2}-1 .
\end{array}\right.
$$

Thus, it is only necessary to compute $g_{n}$, the Fourier coefficients of $G(x)$.

\section{Fast Fourier transforms}

The numerical implementation of computing $g_{n}$ by (19a) is given in this subsection. Let us introduce Fourier collocation points

$$
x_{j}=\frac{2 \pi j}{N}, \quad j=0,1,2, \ldots, N-1 .
$$

For convenience of discussion, denote the following Fourier series approximations

$$
\begin{aligned}
& \bar{G}(x)=\sum_{n=-N / 2}^{N / 2-1} \bar{g}_{n} e^{-i n x}, \\
& J_{0}\left(2 \kappa a\left|\sin \frac{x}{2}\right|\right)=\sum_{n=-N / 2}^{N / 2-1} p_{n} e^{-i n x} .
\end{aligned}
$$

The coefficients of these expansions are computed by FFT (backward in the usual sense) as follows

$$
\begin{aligned}
& \bar{g}_{n}=\frac{1}{N} \sum_{j=0}^{N-1} \bar{G}\left(x_{j}\right) e^{i n x_{j}}, \\
& p_{n}=\frac{1}{N} \sum_{j=0}^{N-1} J_{0}\left(2 \kappa a\left|\sin \frac{x_{j}}{2}\right|\right) e^{i n x_{j},}
\end{aligned}
$$

in which $\bar{G}\left(x_{j}\right)$ is computed by (19). For the value of $\bar{G}(x)$ at $x=0$, the following limit, obtained from (17), can be used

$$
\bar{G}(0)=-\frac{\gamma}{2 \pi}+\frac{i}{4} .
$$

In addition, we denote (18) as

$$
\ln \left(\kappa a\left|\sin \frac{x}{2}\right|\right)=\sum_{n=-\infty}^{\infty} a_{n} e^{-i n x},
$$

where $a_{0}=\ln (\kappa a / 2)$ and $a_{n}=-1 / 2|n|$ for $n \neq 0$. 
Then, by (19a), the Fourier coefficients of $G(x)$ are computed as

$$
g_{n}=\bar{g}_{n}-\frac{1}{2 \pi} u_{n},
$$

where $u_{n}$ is the convolution sum

$$
u_{n}=\sum_{m=-N / 2}^{N / 2-1} p_{m} a_{n-m} \text {. }
$$

We note that the convolution sums in (24) require $N$ multiplications for each $u_{n}$. Thus, the total operations for the convolution sums are of order $O\left(N^{2}\right)$. This cost can be reduced considerably to $O\left(N \log _{2} N\right)$ by the use of a pseudospectral transformation method with dealiasing techniques. ${ }^{7,8}$ For completeness, evaluation of (24) with a "padding" dealiasing technique is given in the Appendix.

\section{SPECTRAL METHOD FOR NEUMANN BOUNDARY CONDITIONS}

We now discuss the Fourier spectral method for the boundary integral equation ( $8 \mathrm{~b})$ of the Neumann boundary conditions. Upon substituting the truncated Fourier series of the layer distribution function $f(\theta)$ into (8b), we get

$$
\begin{gathered}
\frac{i \eta}{2} \sum_{n=-N / 2}^{N / 2-1} f_{n} e^{i n \theta^{\prime}}+\sum_{n=-N / 2}^{N / 2-1}\left[f_{n} \int_{0}^{2 \pi}\left(\frac{\partial^{2} G}{\partial \mathbf{n}^{\prime} \partial \mathbf{n}}-i \eta \frac{\partial G}{\partial \mathbf{n}^{\prime}}\right)\right. \\
\left.\times e^{i n \theta} a d \theta\right]=\sum_{n=-N / 2}^{N / 2-1} b_{n} e^{i n \theta^{\prime}},
\end{gathered}
$$

where $b_{n}$ are the Fourier coefficients of the specified Neumann boundary condition.

Again, the integral appearing in Eq. (25) is directly related to the Fourier coefficients of $\partial^{2} G / \partial \mathbf{n}^{\prime} \partial \mathbf{n}$ and $\partial G / \partial \mathbf{n}^{\prime}$. It is easy to find that the Fourier coefficients of $\partial G / \partial \mathbf{n}^{\prime}$ are the same as those of $\partial G / \partial \mathrm{n}$, already given in the previous section as $h_{n}$. The apparent difficulty here is with the second normal derivative of the Green's function $\partial^{2} G / \partial \mathbf{n}^{\prime} \partial \mathbf{n}$. It can be shown that this function is strongly singular at $x=0$ and, indeed, is not integrable in the ordinary sense. Fortunately, it can also be shown that the integral with the second normal derivative can be transformed into one involving tangential derivatives with reduced singularity. In particular, we have ${ }^{10}$

$$
\begin{aligned}
\int_{0}^{2 \pi} \frac{\partial^{2} G}{\partial \mathbf{n}^{\prime} \partial \mathbf{n}} e^{i n \theta} a d \theta= & \int_{0}^{2 \pi}\left[\frac{1}{a} \frac{\partial e^{i n \theta}}{\partial \theta} \frac{1}{a} \frac{\partial G}{\partial \theta^{\prime}}\right. \\
& \left.+\kappa^{2} \mathbf{n}^{\prime} \cdot \mathbf{n} G e^{i n \theta}\right] a d \theta,
\end{aligned}
$$

where $(1 / a)(\partial / \partial \theta)$ and $(1 / a)\left(\partial / \partial \theta^{\prime}\right)$ represent tangential derivatives on the boundary.

The right-hand side of (26) is now integrable in the sense of the Cauchy principal value. To show this, we only need to note that by the expression of the Green's function given in (6) we get

$$
\begin{aligned}
\frac{\partial G}{\partial \theta^{\prime}} & =-\frac{i}{4} \frac{\partial}{\partial x} H_{0}^{(1)}\left(2 \kappa a\left|\sin \frac{x}{2}\right|\right) \\
& =\frac{i \kappa a}{2} H_{1}^{(1)}\left(2 \kappa a\left|\sin \frac{x}{2}\right|\right) \frac{\partial}{\partial x}\left|\sin \frac{x}{2}\right| \\
& =\frac{i \kappa a}{8} H_{1}^{(1)}\left(2 \kappa a\left|\sin \frac{x}{2}\right|\right) \frac{\sin x}{|\sin x / 2|}
\end{aligned}
$$

Recalling (20), the asymptotic expression of $\partial G / \partial \theta^{\prime}$ for small $x$ is found as

$$
\begin{aligned}
\frac{\partial G}{\partial \theta^{\prime}}= & \frac{\sin x}{8 \pi|\sin x / 2|^{2}}-\frac{\kappa a}{4 \pi} \ln \left(\kappa a\left|\sin \frac{x}{2}\right|\right) \\
& \times J_{1}\left(2 \kappa a\left|\sin \frac{x}{2}\right|\right) \frac{\sin x}{|\sin x / 2|}+O(x),
\end{aligned}
$$

where $O(x)$ denotes smooth terms of order $x$ and higher.

The singular first term shown above is integrable in the sense of the Cauchy principal value. In fact, we have

$$
\begin{aligned}
\frac{1}{2 \pi} \int_{0}^{2 \pi} \frac{\sin x}{|\sin x / 2|^{2}} e^{i n x} d x \\
\quad= \begin{cases}0, & \text { when } n=0, \\
2 i, & \operatorname{sign}(n) \quad \text { when } n \neq 0 .\end{cases}
\end{aligned}
$$

Upon substituting $x=\theta-\theta^{\prime}$ and equating the coefficients of $e^{i n \theta^{\prime}}$, Eq. (25) is reduced to

$$
\begin{aligned}
& \frac{i \eta}{2} f_{n}+f_{n} \int_{0}^{2 \pi}\left(\frac{i n}{a^{2}} \frac{\partial G}{\partial \theta^{\prime}}(x)+\kappa^{2} \cos (x) G(x)-i \eta \frac{\partial G}{\partial \mathbf{n}^{\prime}}(x)\right) \\
& \times e^{i n x} a d x=b_{n},
\end{aligned}
$$

in which we have used the fact that, for a circular cylinder

$$
\mathbf{n}^{\prime} \cdot \mathbf{n}=\cos \left(\theta-\theta^{\prime}\right) .
$$

The integral in (30) will now be evaluated through the Fourier coefficients of each term.

For the first term, the Fourier coefficients of $\partial G / \partial \theta^{\prime}$ are obtained from the relation

$$
\frac{\partial G}{\partial \theta^{\prime}}=-\frac{\partial G}{\partial x}=\sum_{n=-N / 2}^{N / 2-1} \text { in } g_{n} e^{-i n x},
$$

where $g_{n}$ are the Fourier coefficients of $G(x)$ by (13).

The Fourier series approximation of the second term in the integral of (30) can also be found using $g_{n}$ since we have

$$
\cos (x) G(x)=\cos (x) \sum_{n=-N / 2}^{N / 2-1} g_{n} e^{-i n x} \approx \sum_{n=-N / 2}^{N / 2-1} \tilde{g}_{n} e^{-i n x}
$$

where 
TABLE I. Values of the layer distribution function $f(\theta)$ at selected points on the boundary. Dirichlet boundary condition.

\begin{tabular}{rcccc}
\hline \hline$N$ & $\theta=0^{\circ}$ & $\theta=90^{\circ}$ & $\theta=180^{\circ}$ & Error \\
\hline & & $\kappa a=1$ & & \\
4 & 1.101447573 & 1.102982967 & 1.124378820 & $10^{-2}$ \\
8 & 1.113205176 & 1.095419894 & 1.146430615 & $10^{-3}$ \\
16 & 1.112753432 & 1.094877536 & 1.145739275 & $10^{-8}$ \\
24 & 1.112753420 & 1.094877525 & 1.145739263 & $10^{-12}$ \\
& & $\kappa a=10$ & & \\
24 & 4.590213453 & 6.904710445 & 5.180354736 & $10^{-2}$ \\
32 & 4.546357630 & 6.901732036 & 5.132718905 & $10^{-3}$ \\
48 & 4.545461066 & 6.901500667 & 5.132515158 & $10^{-8}$ \\
56 & 4.545461055 & 6.901500659 & 5.132515156 & $10^{-12}$ \\
& & & & \\
224 & & $\kappa a=100$ & & $10^{-3}$ \\
256 & 20.64255659 & 6.841653547 & 18.93255934 & $10^{-9}$ \\
512 & 20.64325731 & 6.842244857 & 18.93221646 & $10^{-12}$ \\
\hline \hline
\end{tabular}

TABLE II. Values of the scattered function $\phi$ at selected points at far-field $r=10 a$. Dirichlet boundary condition.

\begin{tabular}{rcccc}
\hline \hline$N$ & $\theta=0^{\circ}$ & $\theta=90^{\circ}$ & $\theta=180^{\circ}$ & Error \\
\hline & & $\kappa a=1$ & & \\
4 & 0.4146449903 & 0.2787718545 & 0.1852248716 & $10^{-2}$ \\
8 & 0.4224209076 & 0.2612785029 & 0.2551151985 & $10^{-4}$ \\
16 & 0.4224153154 & 0.2613031445 & 0.2552183381 & $10^{-10}$ \\
Exact & 0.4224153154 & 0.2613031445 & 0.2552183381 & $\cdots$ \\
& & $\kappa a=10$ & & \\
24 & 0.8255952003 & 0.1969679200 & 0.1864749710 & $10^{-2}$ \\
32 & 0.8285176644 & 0.1953580665 & 0.2300067055 & $10^{-4}$ \\
48 & 0.8285110664 & 0.1953543814 & 0.2300939707 & $10^{-10}$ \\
Exact & 0.8285110664 & 0.1953543814 & 0.2300939707 & $\cdots$ \\
& & $\kappa a=100$ & & \\
224 & 0.8562228283 & 0.1881301853 & 0.2295232548 & $10^{-3}$ \\
256 & 0.8562289911 & 0.1881326409 & 0.2294229274 & $10^{-10}$ \\
Exact & 0.8562289911 & 0.1881326409 & 0.2294229274 & $\cdots$ \\
\hline \hline
\end{tabular}

TABLE III. Values of the layer distribution function $f(\theta)$ at selected points on the boundary. Neumann boundary condition.

\begin{tabular}{rcccc}
\hline \hline$N$ & $\theta=0^{\circ}$ & $\theta=90^{\circ}$ & $\theta=180^{\circ}$ & Error \\
\hline & & $\kappa a=1$ & & \\
4 & 1.035182633 & 0.3028073027 & 0.8616587030 & $10^{-1}$ \\
8 & 1.200134116 & 0.3972281648 & 0.8518411247 & $10^{-2}$ \\
16 & 1.199187560 & 0.3963806796 & 0.8495643896 & $10^{-7}$ \\
24 & 1.199187560 & 0.3963806589 & 0.8495643587 & $10^{-12}$ \\
& & & & \\
24 & 0.6004486353 & 0.4814454225 & 1.362228889 & $10^{-1}$ \\
32 & 0.6274625969 & 0.6575899642 & 1.577833267 & $10^{-2}$ \\
48 & 0.6302381163 & 0.6567081358 & 1.460119442 & $10^{-7}$ \\
56 & 0.6302381517 & 0.6567081198 & 1.460119455 & $10^{-12}$ \\
& & $\kappa a=100$ & & \\
224 & 0.2185547081 & 1.282490390 & 2.054272775 & $10^{-2}$ \\
256 & 0.2157948725 & 1.283008634 & 2.057912965 & $10^{-7}$ \\
512 & 0.2157947803 & 1.283008643 & 2.057913072 & $10^{-12}$ \\
\hline \hline
\end{tabular}


TABLE IV. Values of the scattered function $\phi$ at selected points at far field $r=10 a$. Neumann boundary condition.

\begin{tabular}{ccccc}
\hline \hline$N$ & $\theta=0^{\circ}$ & $\theta=90^{\circ}$ & $\theta=180^{\circ}$ & Error \\
\hline & & $\kappa a=1$ & & \\
4 & 0.1583300606 & 0.1690204144 & 0.1619964200 & $10^{-1}$ \\
8 & 0.1732916160 & 0.1563414831 & 0.2312523394 & $10^{-4}$ \\
16 & 0.1733358919 & 0.1563260243 & 0.2313583724 & $10^{-10}$ \\
Exact & 0.1733358919 & 0.1563260243 & 0.2313583724 & $\cdots$ \\
& & $\kappa a=10$ & & \\
24 & 0.7679584467 & 0.2167643382 & 0.1574136977 & $10^{-1}$ \\
32 & 0.7740714632 & 0.1956069424 & 0.2282238894 & $10^{-3}$ \\
48 & 0.7740874173 & 0.1955960691 & 0.2283394143 & $10^{-10}$ \\
Exact & 0.7740874173 & 0.1955960691 & 0.2283394143 & $\cdots$ \\
& & $\kappa a=100$ & & \\
224 & 0.7688015277 & 0.1871656432 & 0.2295250315 & $10^{-3}$ \\
256 & 0.7688018590 & 0.1871717295 & 0.2293995512 & $10^{-10}$ \\
Exact & 0.7688018590 & 0.1871717295 & 0.2293995512 & $\cdots$ \\
\hline \hline
\end{tabular}

$$
\bar{g}_{n}=\left\{\begin{array}{l}
\frac{1}{2} g_{-N / 2+1}, \quad n=-N / 2, \\
\frac{1}{2}\left(g_{n-1}+g_{n+1}\right), \quad-N / 2+1 \leqslant n \leqslant N / 2-2, \\
\frac{1}{2} g_{N / 2-2}, \quad n=N / 2-1 .
\end{array}\right.
$$

Hence, Eq. (30) is reduced to the following algebraic equations

$$
\frac{i \eta}{2} f_{n}+2 \pi a f_{n}\left(-\frac{n^{2}}{a^{2}} g_{n}+\kappa^{2} \tilde{g}_{n}-i \eta h_{n}\right)=b_{n},
$$

for $-N / 2 \leqslant n \leqslant N / 2-1$.
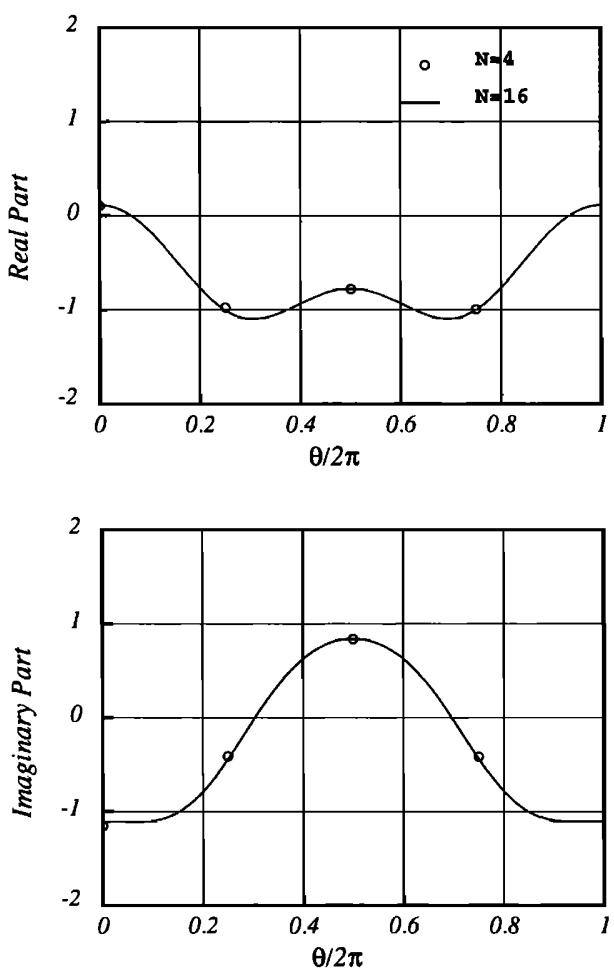

FIG. 1. Layer distribution function $f(\theta)$ for $\kappa a=1$, Dirichlet boundary condition.
Therefore, the Fourier coefficients of the layer distribution function $f(\theta)$ for the Neumann boundary conditions are obtained explicitly as

$$
f_{n}=\frac{b_{n}}{i \eta / 2+2 \pi a\left[-\left(n^{2} / a^{2}\right) g_{n}+\kappa^{2} \tilde{g}_{n}-i \eta h_{n}\right]},
$$

where $g_{n}, \tilde{g}_{n}$, and $h_{n}$ are computed by (23), (33), and (21), respectively.

We point out, however, that $\tilde{g}_{n}$ as given by (33) and, indeed, $h_{n}$ of (21), are not exact for $n=-N / 2$ and $N / 2-1$, owing to a truncated series of $G(x)$ in the computation. Whereas it is possible to compute these two coefficients ex-
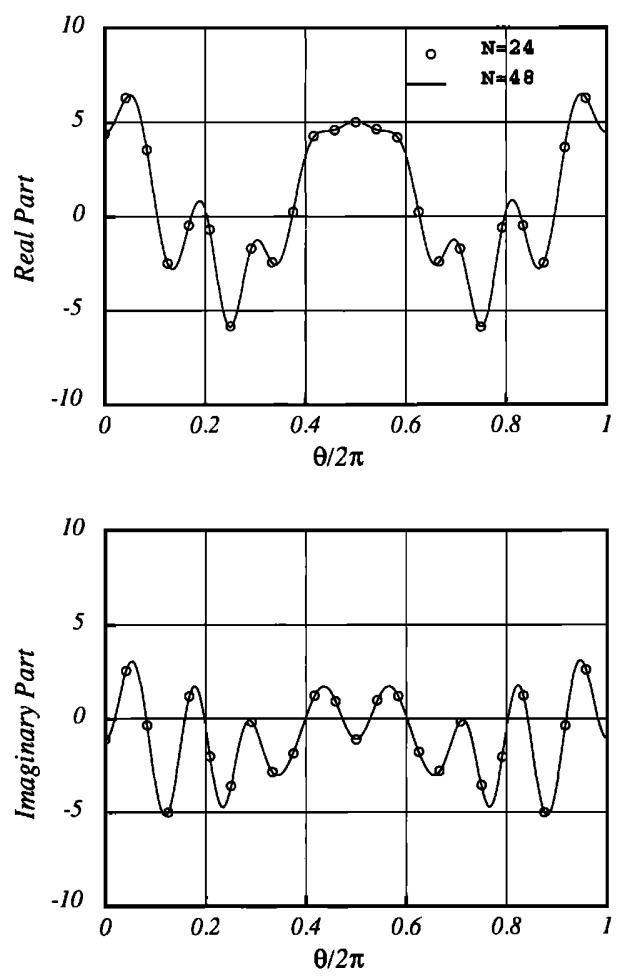

FIG. 2. Layer distribution function $f(\theta)$ for $\kappa a=10$, Dirichlet boundary condition. 

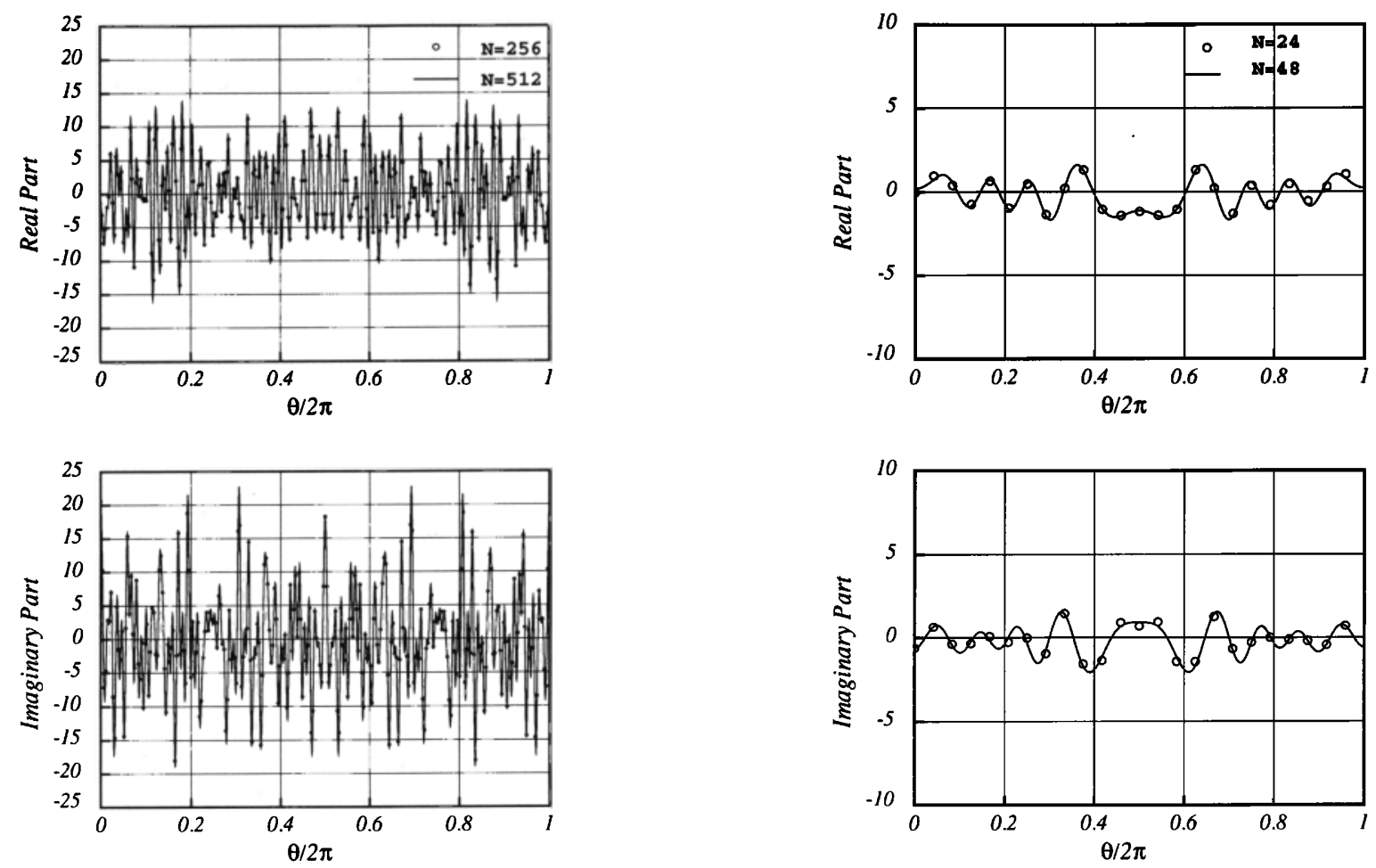

FIG. 3. Layer distribution function $f(\theta)$ for $\kappa a=100$, Dirichlet boundary condition.

FIG. 5. Layer distribution function $f(\theta)$ for $\kappa a=10$, Neumann boundary condition.
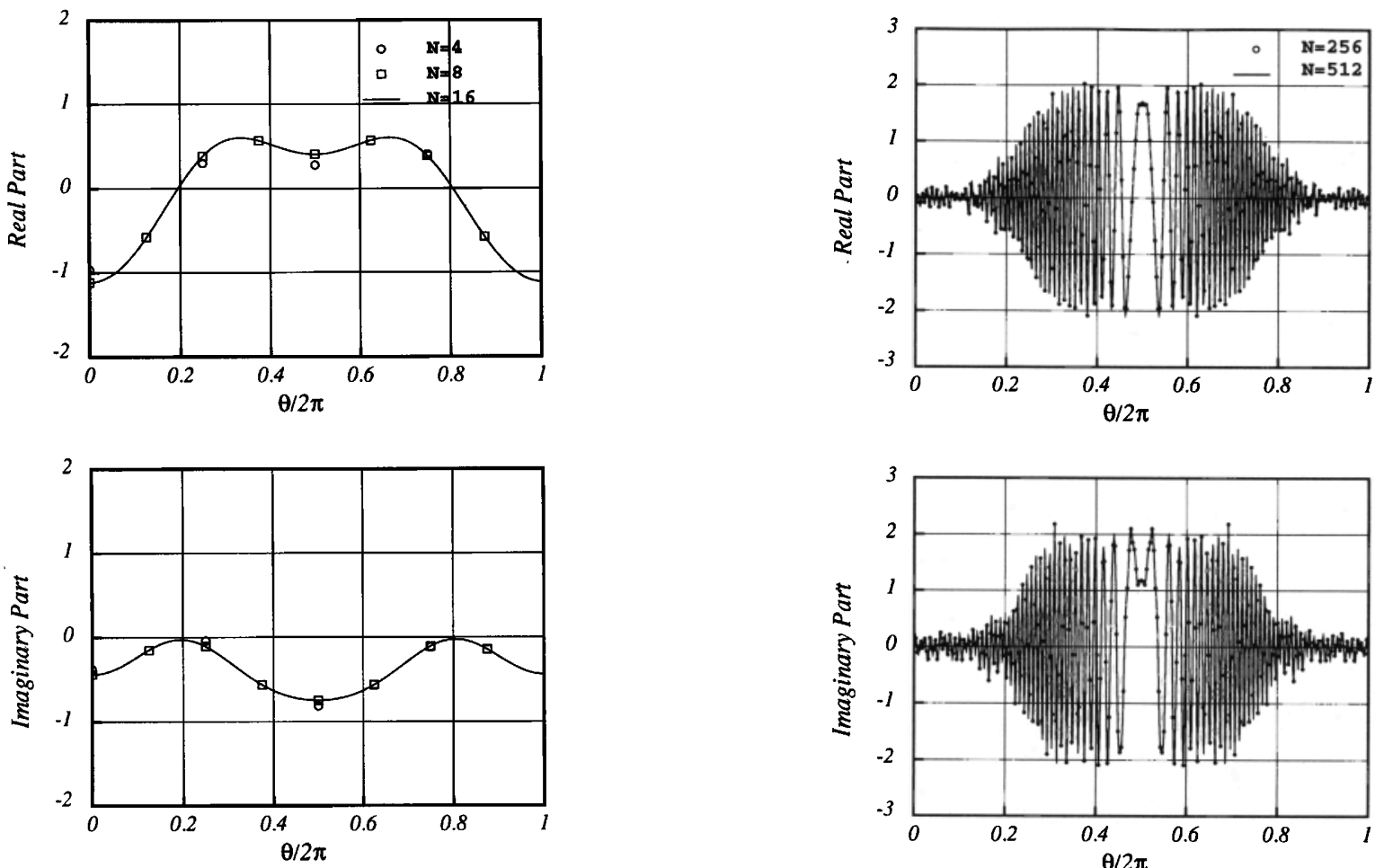

FIG. 4. Layer distribution function $f(\theta)$ for $\kappa a=1$, Neumann boundary condition.

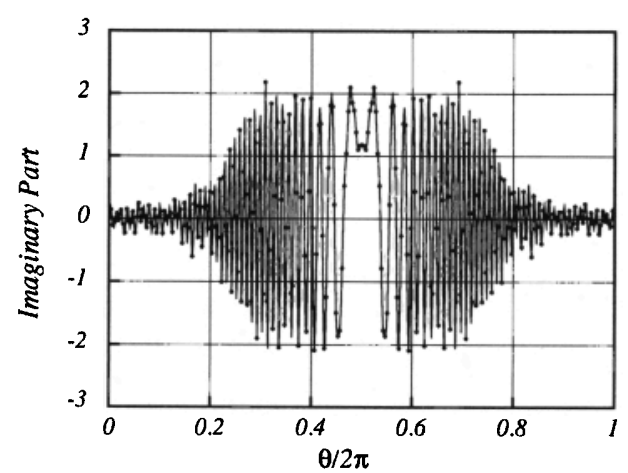

FIG. 6. Layer distribution function $f(\theta)$ for $\kappa a=100$, Neumann boundary condition. 

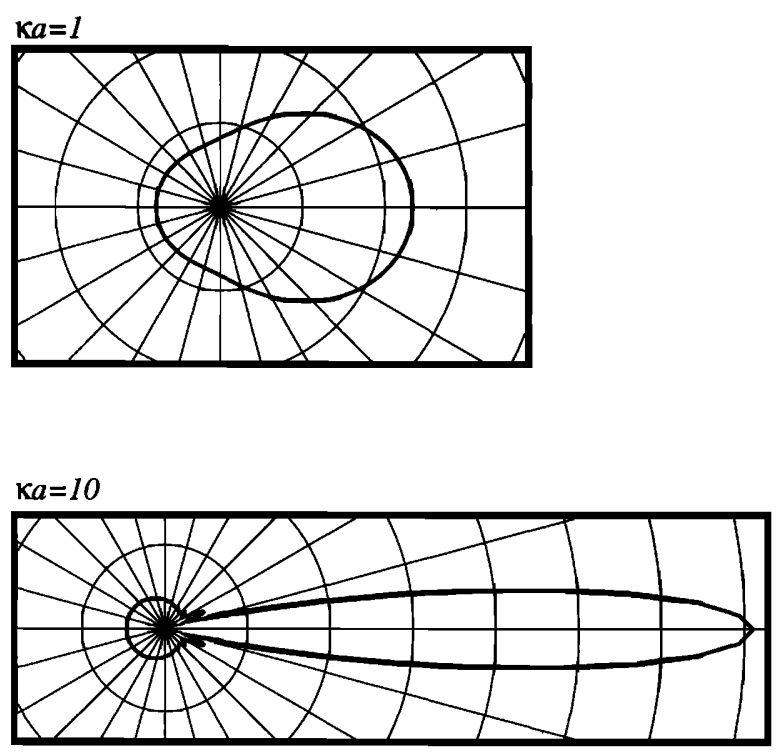

FIG. 7. Directivities of the far-field scattered function, Dirichlet boundary condition.

actly, the resulting error in the last two coefficients of $f_{n}$ is negligible because $b_{n}$, in the numerator, decays exponentially as for smooth boundary conditions. That is, $f_{n}$ for $n=$ $-N / 2$ and $N / 2-1$ are necessary negligibly small if $N$ is sufficiently large. For simplicity and practicality, (21) and (33) are retained in the numerical calculations.

\section{NUMERICAL EXAMPLES}

In this section, numerical results of a plane-wave scattering by a circular cylinder are presented. The incident wave is assumed to be

$$
\phi_{i}=e^{i \kappa x}
$$
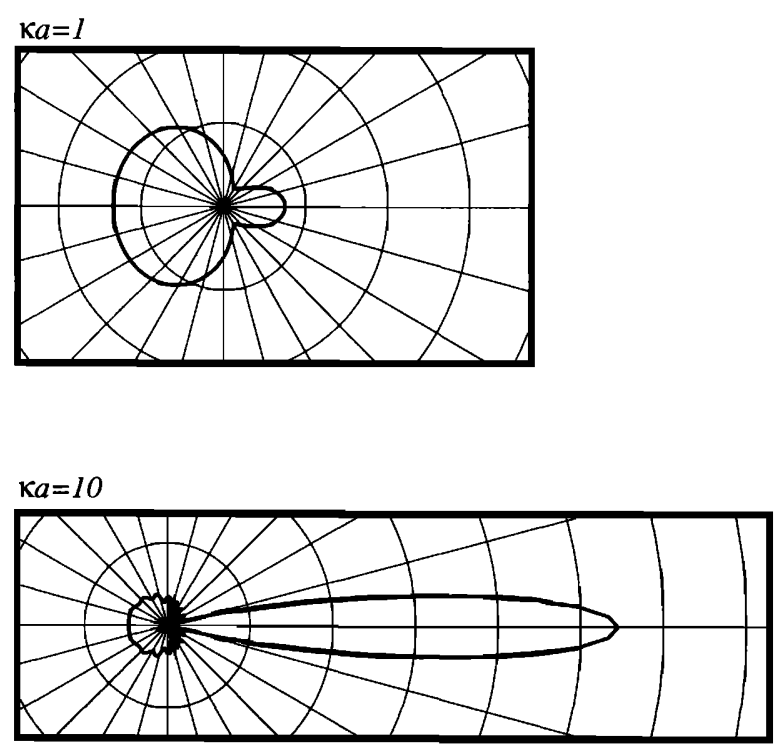

FIG. 8. Directivities of the far-field scattered function, Neumann boundary condition.
The scattered wave $\phi$ satisfies the Helmholtz Eq. (1). The boundary conditions considered here are the Dirichlet type $\phi=-\phi_{i}$ and the Neumann type $\partial \phi / \partial \mathbf{n}=-\partial \phi_{i} / \partial \mathrm{n}$.

The solutions for the scattered field are obtained by the layer representation (2) as

$$
\begin{aligned}
\phi\left(\mathbf{r}^{\prime}\right) & =\int_{0}^{2 \pi}\left(\frac{\partial G}{\partial \mathbf{n}}-i \eta G\right) f(\theta) a d \theta \\
& =a \sum_{n=-N / 2}^{N / 2-1} f_{n} \int_{0}^{2 \pi}\left(\frac{\partial G}{\partial \mathbf{n}}-i \eta G\right) e^{i n \theta} d \theta .
\end{aligned}
$$

The above integral can be easily evaluated directly using FFT, since the Green's function has no singularity for points lying outside of the boundary. The details are omitted here.

For plane incident waves, an exact solution is given by infinite series of the Bessel and Hankel functions. ${ }^{6}$ Our purpose here is to demonstrate the exponential rate of convergence of the numerical solutions. We emphasize again that the numerical formulation applies to any form of the incident waves. Due to its simplicity, a sample FORTRAN program is listed in the Appendix.

In numerical calculations, the radius of the cylinder $a$ is taken to be 1 and also $\eta=1$. Computations for $\kappa a=1,10$, and 100 have been carried out. In Tables I-IV, numerical values of the layer distribution function $f(\theta)$ and the scattered function $\phi$ at far field are given for selected points in space. Exact values at far field are also shown in the tables. Clearly, as the number of Fourier collocation points increases, the numerical solution converges exponentially fast. Significant improvements in accuracy are observed with relatively small increase of the number of data points. This is often true for spectral methods in general. The error decreases dramatically when the number of points is large enough to resolve the basic features of the solution.

The corresponding layer distribution function $f(\theta)$ is plotted in Figs. 1-6 for the Dirichlet and Neumann boundary conditions for $\kappa a=1,10$, and 100 . These graphs demonstrate again the remarkable accuracy of the Fourier spectral methods with relatively small number of datum points.

Far-field scattered intensities, computed as $|\mathbf{r}| \phi^{2}$, are plotted in Figs. 7 and 8 for the Dirichlet and Neumann boundary conditions, respectively.

\section{CONCLUSIONS}

A fast numerical solution of wave scattering by a circular cylinder has been presented. It is shown that by properly removing the nonsmoothness of the integral kernels of the boundary integral equations, spectrally accurate numerical solutions are obtained. The numerical error decays exponentially as the number of datum points increase. This implies that the present method requires significantly fewer points for achieving a given accuracy in comparison with previous numerical approaches. The present method is also easy to implement.

Moreover, the combined single- and double-layer formulation of the boundary integral equations ensures the uniqueness of the numerical solution for all frequencies. It is 
shown that the hypersingularity of the boundary integral equations can be handled easily in the spectral method.

\section{ACKNOWLEDGMENTS}

The author wishes to thank Dr. D. G. Lasseigne for helpful comments and discussions. This work was supported by the National Aeronautics and Space Administration under NASA Contract NAS1-19480 while the author was in residence at the Institute for Computer Applications in Science and Engineering, NASA-Langley Research Center, Hampton, VA 23665.

\section{APPENDIX}

\section{Exact expressions of $\boldsymbol{g}_{\boldsymbol{n}}$ and $\boldsymbol{h}_{\boldsymbol{n}}$}

In this appendix we derive the exact analytic expressions for the Fourier coefficients of $G(x)$ and $(\partial G / \partial \mathrm{n})(x)$.

It can be shown that, e.g., by (7.2.51) of Ref. 6,

$$
H_{0}^{(1)}\left(2 \kappa a\left|\sin \frac{x}{2}\right|\right)=\sum_{m=-\infty}^{\infty} H_{m}^{(1)}(\kappa a) J_{m}(\kappa a) e^{-i m x} \text {. }
$$

Hence

$$
\begin{aligned}
g_{n} & =\frac{1}{2 \pi} \int_{0}^{2 \pi} G(x) e^{i n x} d x \\
& =\frac{1}{2 \pi} \int_{0}^{2 \pi} \frac{i}{4} H_{0}^{(1)}\left(2 \kappa a\left|\sin \frac{x}{2}\right|\right) e^{i n x} d x \\
& =\frac{i}{4} H_{n}^{(1)}(\kappa a) J_{n}(\kappa a) .
\end{aligned}
$$

Moreover, for $n \neq 0$, using integration by part and (A1)

$$
\begin{aligned}
h_{n}= & \frac{1}{2 \pi} \int_{0}^{2 \pi} \frac{\partial G}{\partial \mathbf{n}}(x) e^{i n x} d x \\
= & -\frac{i \kappa}{8 \pi} \int_{0}^{2 \pi} H_{1}^{(1)}\left(2 \kappa a\left|\sin \frac{x}{2}\right|\right)\left|\sin \frac{x}{2}\right| e^{i n x} d x \\
= & \frac{\kappa^{2} a}{8 \pi n} \int_{0}^{2 \pi} H_{0}^{(1)}\left(2 \kappa a\left|\sin \frac{x}{2}\right|\right)\left|\sin \frac{x}{2}\right| \cos \frac{x}{2} e^{i n x} d x \\
= & \frac{\kappa^{2} a}{16 \pi n} \int_{0}^{2 \pi}\left(\sum_{m=-\infty}^{\infty} H_{m}^{(1)}(\kappa a) J_{m}(\kappa a) e^{-i m x}\right) \\
& \times \sin x e^{i n x} d x \\
= & -\frac{\kappa^{2} a i}{16 n}\left[H_{n+1}^{(1)}(\kappa a) J_{n+1}(\kappa a)\right. \\
& \left.-H_{n-1}^{(1)}(\kappa a) J_{n-1}(\kappa a)\right] \\
= & -\frac{\kappa^{2} a}{4 n}\left(g_{n+1}-g_{n-1}\right),
\end{aligned}
$$

where use has been made of the formula ${ }^{9}$

$$
\frac{d}{d z}\left[z H_{1}^{(1)}(z)\right]=z H_{0}^{(1)}(z) .
$$

For $\boldsymbol{n}=\mathbf{0}$, further calculations show that

$$
\begin{aligned}
h_{0}= & \frac{\kappa^{2} a i}{16}\left[H_{2}^{(1)}(\kappa a) J_{2}(\kappa a)-H_{0}^{(1)}(\kappa a) J_{0}(\kappa a)\right] \\
& -\frac{i}{4 a} H_{1}^{(1)}(\kappa a) J_{1}(\kappa a) \\
= & \frac{\kappa^{2} a}{4}\left(g_{2}-g_{0}\right)-\frac{1}{a} g_{1} .
\end{aligned}
$$

\section{Evaluation of convolution sums}

An algorithm of computing convolution sums $u_{n}$ with $O\left(N \log _{2} N\right)$ operations is shown below. ${ }^{8}$

Let $M \geqslant 3 N$ and

$$
\xi_{j}=2 \pi j / M, \quad j=0,1,2, \ldots, M-1 .
$$

Compute the following using FFT for $j=0,1,2, \ldots, M-1$ :

$$
\begin{aligned}
& A_{j}=\sum_{m=-M / 2}^{M / 2-1} \tilde{a}_{m} e^{-i m \xi_{j}}, \\
& P_{j}=\sum_{m=-M / 2}^{M / 2-1} \tilde{p}_{m} e^{-i m \xi_{j}},
\end{aligned}
$$

where

$$
\begin{aligned}
& \tilde{a}_{m}= \begin{cases}a_{m}, & -N \leqslant m \leqslant N-1, \\
0, & \text { other, }\end{cases} \\
& \tilde{p}_{m}= \begin{cases}p_{m}, & -N / 2 \leqslant m \leqslant N / 2-1, \\
0, & \text { other, }\end{cases}
\end{aligned}
$$

and form the product

$$
U_{j}=A_{j} P_{j} .
$$

Then the convolution sum $u_{n}$ is the (backward) FFT of $U_{j}$ as follows

$$
u_{n}=\frac{1}{M} \sum_{j=0}^{M-1} U_{j} e^{i n \xi_{j}},
$$

for $-N / 2 \leqslant n \leqslant N / 2-1$.

\section{FORTRAN program}

A FORTRAN program of implementing the Fourier spectral method is listed below. (The external routines cftti, cfttf, and cfttb denote initializing, forward, and backward FFT transforms, respectively.) 
progran circle c n : number of points; isoft $t$ : Dirichlet B.C.; isoft $=0$ : Ieumann B.C. parameter $(n=32$, ak $=10,0$, isoft $t=1,0$ ta $a=1,0, n 1=n-1, n h a l f=n / 2, m=3 * n$, $>$ rn=float $(n), p i=3.14159266358979324$, ouler $=0.57721566490153286)$ complex $b(0: n 1), f n(0: n 1), g b a r(0: n 1), g n(0: n 1), h n(0: n 1), p(0: n 1)$, $>$ gtilde(0:n1), am(0:n-1),pm(0:m-1), , gsave(2000), gsave2(2000), $\theta i, p h i$ $\theta i=(0.0,1.0)$

call cffti(n, rsavo)

call gotbc $(n, a k, b, \theta i, p i)$

call cfftf $(n, b$, usave)

do $10 \mathrm{j}=0, n-1$

mp=2.0*ak*abs $(\sin (p i * f l o a t(j) / x n))$

if $(j$. .eq. o) the

$\operatorname{gbar}(0)=-$ euler $/ 2.0 / \mathrm{pi}+e \mathrm{i} / 4.0$

$P(0)=1.0$

olse

$\operatorname{gbar}(j)=0 i / 4.0 *($ bes jo $(\operatorname{tmp})+e i *$ besyo $(\operatorname{tmp})$ $+0.6 * a \log (t m p / 2.0) * b e s j 0(t m p) / p i$

(j)

10

continue

call cfftb(n,gbar, vsave)

call cfttb(n,p, vsave)

$\operatorname{am}(0)=\operatorname{alog}(a k / 2.0)$

$\operatorname{am}(2 * n)=-1.0 / 2.0 /$

do $21 \quad i=1, n-1$

$\operatorname{arc}(i)=-1.0 / 2.0 /$ float $(i)$

$21 \quad a n(2 * n+i)=1.0 / 2.0 / 1$ loat $(i-n)$

do $22 i=0$, nhalt -1

$\operatorname{pm}(i)=p(i)$

$22 \quad$ pm $(5 *$ nhal $f+i)=p($ nhal $f+i)$

call cffti (m, zsave2)

call cffte (m, am, vsave2)

call cffte (m, pm, vaave2)

do $23 j=0, m-1$

$23 \quad p m(j)=a n(j) * p n(j)$

call cfftb (m,pm, vsave2)

do $31 \quad i=0$, nhale -1

$g n(i)=g b a r(i)-0.5 * \operatorname{pm}(i) / f l o a t(m) / p i$

$31 \mathrm{gn}($ nhal $f+i)=\operatorname{gbar}($ nhalf $+i)-0.5 * \mathrm{pm}(5 *$ ghalf $+i) / f$ loat $(\mathrm{m}) / \mathrm{p}$

$\ln (0)=\operatorname{ak} * * 2 / 4.0 *(\operatorname{gn}(2)-\operatorname{gn}(0))-\operatorname{gn}(1)$

$h n($ nhalf -1$)=a k * * 2 / 4.0 /$ float (nhall-1) $* \operatorname{gn}($ nhalf -2$)$

hn (nhalf $)=$ ak $* * 2 / 4.0 /$ float (nhalf) $* g n($ nhalf +1$)$

$h n(n-1)=a k * * 2 / 4.0 *(g n(0)-g n(n-2))$

gtildo $(0)=0.5 *(g n(1)+\operatorname{gn}(n-1))$

gtildo (nhalf -1$)=0.5 * \operatorname{gn}($ nhalf -2$)$

gtilde(nhalf $)=0.5 * g n($ nhalf +1$)$

gtilde $(n-1)=0.5 *(g n(0)+g n(n-2))$

do $32 i=1, n-2$

itrue $=1$

if (i.ge.nhalf) itrue $=i-n$

if (i.eq.nhalf-1.or.i.eq.nhalf) go to 32

hn $(i)=-\operatorname{ak} * * 2 / 4.0 /$ float (itrue $) *(g n(i+1)-\operatorname{gn}(i-1))$

gtilde $(i)=0.5 *\left(\operatorname{gn}(i-1)+g^{n}(i+1)\right)$

continue

do $40 \quad i=0, n-1$

if (isott.eq.1) the

$\ln (i)=b(i) /(0.5 * \operatorname{mn}+2.0 * p i *(\ln (i)-\operatorname{oi} * \operatorname{ata} * \operatorname{gn}(i)))$

0180

itrue $=$

if (i.ge. nhalf) itrue $=i-n$

$>$

$\ln (i)=b(i) /(0.5 * 0 i * 0 t a * r n+2.0 * p i *(-1$ loat $($ itrue $) * * 2 * g n(i)$

40 contin

The folloning is to tind phi at far fiold rero

$r 0=10.0$

npoint $=4$

do $70 i i=1$, npoint

$s j=2.0 *$ pi*1loat $(i i-1) / f l o a t$ (npoint)

do $71 j=0, n-1$

thet $a=2.0 *$ pi*float $(j) / r n$

$r j=2 q r t(1.0+r 0 * r 0-2.0 * r 0 * \cos ($ theta $-8 j)$

$d j=1.0-r 0 * \cos$ (theta $-8 j$ )

tap $=a k * r j$

$\operatorname{gn}(j)=0 i / 4.0 *$ (besjo(tmp) + a $i *$ besyo(tmp)

71

call cfftb $(n, g n$, vaave)

call clftb (n,gn, gave

phi $=0.0$

do $72 i=0, n-1$

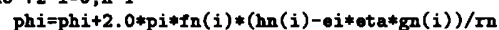

orite $(3,100)$ ro, aj, phi, cabs (phi)

format (' $r 0=$ ', $\bullet 15.6$,' theta $=$ ', $015.6 /$ ' phi=',3017.10)

at op

subroutine gøtbc(n, ak, $b, \bullet i, p i)$

complox $\bullet i, b(0: n-1)$, tmp

do $10 \mathrm{j}=0, \mathrm{n}-1$

tap $=0 i * a k * \cos (2.0 *$ pi*float $(j) / f l o a t(n))$

$b(j)=-\operatorname{cosp}\left(t_{m p}\right)$

end
${ }^{1}$ D. Colton and R. Kress, Integral Equation Methods in Scattering Theory (Wiley-Interscience, New York, 1983).

${ }^{2}$ C. A. Brebbia and M. S. Ingber, Boundary Element Technology VII (Elsevier, New York, 1992).

${ }^{3}$ N. N. Bojarski, "Scattering by a cylinder: A fast exact numerical solution," J. Acoust. Soc. Am. 75, 320-323 (1984).

${ }^{4} \mathrm{G}$. T. Schuster, "A fast exact numerical solution for the acoustic response of concentric cylinders with penetrable interfaces," J. Acoust. Soc. Am. 87, 495-502 (1990).

${ }^{5}$ G. Chen and J. Zhou, Boundary Element Methods (Academic, New York, 1992).

${ }^{6} \mathrm{P}$. Morse and H. Feshbach, Methods of Theoretical Physics (McGraw-Hill, New York, 1953).

${ }^{7}$ D. Gottlieb and S. Orszag, Numerical Analysis of Spectral Methods: Theory and Applications, SIAM, 1977.

${ }^{8}$ C. Canuto, M. Y. Hussaini, A. Quarteroni, and T. A. Zang, Spectral Methods in Fluid Dynamics (Springer-Verlag, Berlin, 1988).

${ }^{9}$ M. Abramowitz and I. A. Stegun, Handbook of Mathematical Functions (Dover, New York, 1965).

${ }^{10}$ A. J. Burton and G. F. Miller, "The application of integral equation methods to the numerical solution of some exterior boundary-value problems," Proc. R. Soc. London Ser. A 323, 201-210 (1971). 